\title{
The Implications of Program Genres for the Design of Social Television Systems
}

\author{
David Geerts \\ CUO, IBBT/K.U.Leuven \\ Parkstraat 45 Bus 3605 \\ 3000 Leuven, Belgium \\ +3216323195 \\ david.geerts@soc.kuleuven.be
}

\author{
Pablo Cesar \\ CWI \\ Kruislaan 413, Amsterdam \\ 1098 SJ, The Netherlands \\ +3120 5929332 \\ p.s.cesar@cwi.nl
}

\author{
Dick Bulterman \\ CWI \\ Kruislaan 413, Amsterdam \\ 1098 SJ, The Netherlands \\ +31205929332 \\ dick.bulterman@cwi.nl
}

\begin{abstract}
In this paper, we look at how television genres can play a role in the use of social interactive television systems (social iTV). Based on a user study of a system for sending and receiving enriched video fragments to and from a range of devices, we discuss which genres are preferred for talking while watching, talking about after watching and for sending to users with different devices. The results show that news, soap, quiz and sport are genres during which our participants talk most while watching and are thus suitable for synchronous social iTV systems. For asynchronous social iTV systems film, news, documentaries and music programs are potentially popular genres. The plot structure of certain genres influences if people are inclined to talk while watching or not, and to which device they would send a video fragment. We also discuss how this impacts the design and evaluation of social iTV systems.
\end{abstract}

\section{Categories and Subject Descriptors}

H.5.1 [Information Interfaces and Presentation]: Multimedia Information Systems, J.7 [Computers in Other Systems]: Consumer Products, H.4.3 [Information Systems Applications]: Communications Applications

\section{General Terms}

Measurement, Design, Experimentation, Human Factors

\section{Keywords}

Social Interactive Television, Television Genre, Sharing Video

\section{INTRODUCTION}

A main feature of most social interactive television systems is the support of communication while watching television. These systems usually intend to recreate the social use of traditional television as conversation starter [25], but for several remotely located viewers rather than for collocated viewers. Users can either communicate by voice or using text, but this communication does not necessarily have to be synchronous and can be asynchronous as

Permission to make digital or hard copies of all or part of this work for personal or classroom use is granted without fee provided that copies are not made or distributed for profit or commercial advantage and that copies bear this notice and the full citation on the first page. To copy otherwise, or republish, to post on servers or to redistribute to lists, requires prior specific permission and/or a fee.

uxTV'08, October 22-24, 2008, Silicon Valley, California, USA.

Copyright 2008 ACM 978-1-60558-100-2/08/10...\$5.00. well, e.g. by sending recommendations or leaving notes for people who will watch the same content at another time [19]. Some systems allow users to share video fragments with each other, making annotations on them to add a personal note [8].

Research on social interactive television has been focused on the creation of the systems itself (e.g. [19]), or on specific features such as a comparison between voice chat and text chat [16]. Several user studies have indicated that television program genres play an important role in the use of social iTV (e.g. [18]), especially regarding attention and communication activity. However, research into why and how certain genres have an impact on the use of social iTV systems remains scarce. With this paper, we want to contribute to the design of social interactive television by providing more insight into the role of television program genres in social iTV, in order to improve the success and effectiveness of these systems.

The system we used in an extensive user study for tackling this issue is the Ambulant Annotator [4], which allows its users to send and receive enriched video fragments from and to a number of devices, including TV, PC and mobile phones. This range of devices also raises questions about the impact on users of several devices to receive and watch content and the role of program genres in this respect.

In this paper, we will review related work regarding video sharing, diverse devices and television genres. After a description of the system and the user study we set up, we will then present the results regarding our two main research issues: on which devices do people prefer to receive and watch video content; and which television program genres play a role in communicating about and sharing video content. In the discussion section, we will bring the results together and interpret them, and finally we will draw some main conclusions regarding the design of social iTV systems.

\section{RELATED WORK}

As described above, social interactive television comes in different flavors. As our system provides the user with different devices as primary or secondary screen and allows the user to share video content, we will first review work on video sharing systems and device divergence. We will end this section with a look at how television genres have been studied in the past.

\subsection{Video sharing systems}

Systems allowing users to share audiovisual material can be divided into four categories: online video sharing, WebTV, interactive television, and enriched instant messaging. 
Lately, online video sharing has become a primary activity in the World Wide Web. Some clear examples include YouTube ${ }^{1}$, Yahoo! Videos ${ }^{2}$, and MySpace ${ }^{3}$. These systems provide the user easy-to-use interfaces for uploading, searching, viewing, rating, and recommending videos. In this paper we are especially interested in the interface for recommending video content, which normally is performed by sending an e-mail to the video recipient or by embedding the video into a social web site such as Facebook ${ }^{4}$. A number of research papers have focused on video sharing. One of them [17], for example, studies the usage of popular online video sharing systems and categorizes the most popular and downloaded video genres.

While online video sharing systems are, in theory, intended for user-generated content, currently a number of WebTV systems such as Joost ${ }^{5}$ and Lycos Cinema ${ }^{6}$ are becoming popular as well. These WebTV solutions, similar to IPTV systems, are targeted for watching television programs using the Internet infrastructure. From this paper's perspective the most interesting functionality, apart from recommending videos, is to watch television together across distances. Similar solutions exist in the form of Peer-to-Peer television such as Tribler ${ }^{7}$.

At the same time, traditional interactive television systems are upgrading their infrastructure in order to provide social activities. We can term such solutions as social interactive television. For example, CollaboraTV from AT\&T [19] permits to record the viewer's comments while watching a television program. Then, such comments are replayed when a friend is watching the video using avatars to identify who has said what. Synchronous solutions, such as Motorola's SocialTV [18] and Alcatel's AmigoTV [9], have been proposed as well. Several user studies focus on synchronized communication while watching media [18], [32] and asynchronous communication of enriched media material [33], [7].

While media-based systems are starting to provide communication and sharing functionality, communication systems are starting to provide media capabilities. For example, enriched instant messaging solutions such as Messenger $\mathrm{TV}^{8}$ from Microsoft and Zync! ${ }^{9}$ from Yahoo! now provide the option of sharing synchronized videos while chatting.

Research into these systems has just started and still many research questions remain to be answered. In terms of user studies, there is a need to understand which genres and video formats are better suited for social activities such as sharing, commenting, or watching across distances. Moreover, we should understand how to better make use of the devices that are surrounding users in a specific moment. In terms of infrastructure, there is a need for systems that allow the user to identify a fragment of a video to be shared. Furthermore, the user can be provided with interfaces for

\footnotetext{
${ }^{1} \mathrm{http}: / / \mathrm{www}$. youtube.com/

${ }^{2}$ http://video.yahoo.com/

${ }^{3} \mathrm{http}: / / \mathrm{www} . \mathrm{myspace} . \mathrm{com} /$

${ }^{4}$ http://www.facebook.com/

${ }^{5} \mathrm{http}: / /$ www.joost.com/

${ }^{6} \mathrm{http}: / /$ cinema.lycos.com/

${ }^{7} \mathrm{http}: / /$ www.tribler.org/

${ }^{8} \mathrm{http}: / /$ messengertv.msn.com/mkt/en-gb/default.htm

${ }^{9}$ http://timetags.research.yahoo.com/zync/
}

enriching the identified fragment before sharing it. Finally, we need to provide solutions for device interoperability, so different devices (e.g., television, laptop, mobile phone) can be used together depending on the contextual situation of the user.

\subsection{Device divergence}

As illustrated in the previous section, current solutions for sharing media tend to be designed in a monolithic manner: the user can choose either online video sharing systems, or WebTV, or social interactive television, or enhanced instant messaging solutions. On the other hand, we believe that a key challenge is to dynamically distribute media content rendering and media control capabilities across the most suitable device surrounding the user in a specific moment. At some times, the user might want to watch video material in a television screen or through a projector connected to his/her laptop. But at other moments, for example when on the move, the user would like to render video clips in his/her mobile device. Similar requirements can be found in current research literature. It is clear that multimedia consumers are becoming mobile [26]. Moreover, recent findings state that 'our devices should collaborate to support a notion of user-centric activities that span multiple devices' [11]. Finally, O'Hara et al. [27] report that users found it frustrating to get video material in mobile devices and conclude that a better integration among devices is needed.

These findings contradict the classic view of device convergence that has been stated several times in the past, and which is also challenged by Cardoso [5]. Cardoso states that rather than focus on interactive television, where all interaction is handled by one device (the television), we should focus on networked television. The latter consists of a number of devices that connect with each other to make interaction and communication possible. For example, a popular way of interacting currently with television is by SMS voting or texting, indicating that users see no problem using several devices to multitask with various technologies in a network.

Figure 1 illustrates the characteristics of the devices that can be used for viewing and sharing digital videos. The table differentiates between shared/private devices and elaborates on the different usages each of the devices is intended for. For example, the television is a shared device with high rendering capabilities, intended for entertainment, and is normally used as a primary screen. The iPod is a private device with a small screen that can be used as a secondary screen for television watching, and might provide some sharing of videos capabilities.

\begin{tabular}{|c|c|c|c|c|c|c|}
\hline & TV & $\begin{array}{l}\text { Remote } \\
\text { Control }\end{array}$ & $\begin{array}{c}\text { Apple } \\
\text { iPod }\end{array}$ & $\begin{array}{c}\text { Nokia } \\
\text { N770 }\end{array}$ & 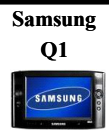 & $\square$ \\
\hline $\begin{array}{l}\text { Social } \\
\text { Experience }\end{array}$ & Shared & Shared & Private & Private & Private & Private \\
\hline $\begin{array}{l}\text { Viewing / } \\
\text { Feedback } \\
\text { Experience }\end{array}$ & HD Screen & $\begin{array}{c}\text { None } \\
\text { (onscreen) }\end{array}$ & $\begin{array}{l}\text { Small } \\
\text { screen }\end{array}$ & $\begin{array}{l}\text { Small } \\
\text { screen }\end{array}$ & $\underset{\text { screen }}{\text { Medium }}$ & $\begin{array}{c}\text { Big } \\
\text { Screen }\end{array}$ \\
\hline $\begin{array}{l}\text { Authoring } \\
\text { Experience }\end{array}$ & None & $\begin{array}{c}\text { Minimal } \\
\text { (onscreen) }\end{array}$ & Medium & Medium & High & $\begin{array}{l}\text { Very } \\
\text { High }\end{array}$ \\
\hline User Attitude & Entertain & Control & $\begin{array}{c}\text { Media } \\
\text { sharing }\end{array}$ & $\begin{array}{c}\text { Media } \\
\text { sharing }\end{array}$ & $\begin{array}{c}\text { Media } \\
\text { sharing }\end{array}$ & Produce \\
\hline $\begin{array}{l}\text { Interaction } \\
\text { Role }\end{array}$ & No & Control & $\begin{array}{c}\text { Control } \\
\text { Video edit }\end{array}$ & $\begin{array}{c}\text { Control } \\
\text { Video edit }\end{array}$ & $\begin{array}{c}\text { Control } \\
\text { Video edit }\end{array}$ & $\begin{array}{c}\text { Video } \\
\text { edit }\end{array}$ \\
\hline $\begin{array}{l}\text { Rendering } \\
\text { Role }\end{array}$ & $\begin{array}{c}\text { Primary } \\
\text { Screen }\end{array}$ & None & $\begin{array}{l}\text { Secondary } \\
\text { Screen }\end{array}$ & $\begin{array}{c}\text { Secondary } \\
\text { Screen }\end{array}$ & $\begin{array}{c}\text { Secondary } \\
\text { Screen }\end{array}$ & $\begin{array}{c}\text { Primary } \\
\text { Screen }\end{array}$ \\
\hline
\end{tabular}

Figure 1: Categorization of Devices at Home 
The Nokia 770 is a tablet-based device similar to the iPod, in which ink and 2D gestures can be used for interaction, while the Samsung Q1 is a ultra-mobile PC with bigger screen and computing power. The two latter devices are used in the Ambulant Annotator system. As the use of some devices may be ambiguous, the qualification used in the table above indicates the normal and primary use of each device.

Lately, research on secondary screens is providing a number of interesting results [6]. Firstly, remote controls that have a screen are often considered unusable and their rendering capabilities could be further exploited [1]. Additionally, it has been found that more and more viewers use their laptop while watching television [31]. Currently, two research areas take advantage of the secondary screen: interactive television learning [14] and content selection [28],[10].

Conversely, mobile devices can be used as the primary screen for video material. While most of the research in this area studies the technological factors and the perceived quality (e.g., [24]), there are also studies that focus on the current usages of mobile devices in the context of watching video. Södergård [34] concludes that people who watch television on mobile devices do this for short periods of time, and the main advantage was considered to be the "anytime, anywhere availability". Repo et. al [29] hint at the importance of the private experience by using a mobile phone to watch videos. Finally, O'Hara et al. provide an extensive study on how and why video material is consumed in mobile devices [27]. They conclude that even though consuming video in mobile devices is a privatizing technology, it might facilitate togetherness in the home as people can watch "their own content while being in proximity to family". Moreover, mobile phones allowed the participants to bring content to social situations and places in which a conventional TV was not available.

There have been several studies on the motives of people to share media material, mostly photographs, using mobile phones [35], [23], [22]. Taylor e.a. [35] observed the social norms around gift-giving and the demand of reciprocity with mobile phones. Another study categorized the uses of photo sharing in two dimensions: social/individual and functional/affective [23]. The results indicate that affective usage was the most predominant. House et al. [22] provide a similar categorization: creating and maintaining social relationships, personal and group memory, self expression, self presentation, and functional. In most of the cases, the usage was mainly incidental and not a planned activity.

In line with these findings, the system used in this study was designed to allow the user to receive video clips in various ways: on the television, on a mobile phone, via e-mail, or by uploading a link of a video on a blog. This leads to a quite important question for current video sharing systems: which device would users prefer for receiving video clips in which context? To our knowledge, there is no research that compares all these sharing channels. Therefore, in this paper we will discuss some results that can give us insight in user preferences for each single device. Furthermore, we want to investigate if program genres also play a role in these user preferences.

\subsection{Sociability of television genres}

The systems discussed above all share the fact that video or television content plays a prominent role. However, this content is not always treated the same way by viewers, as different genres result in a different experience. In television studies, there is a long tradition in studying genres of television content. Even though there is an audience perspective in genre studies, it predominantly looks at what constitutes a genre, how certain television programs fit into a genre, and especially how audiences use genre to understand and enjoy programs [2]. The communication patterns surrounding certain television genres, however, are not explicitly studied in genre studies but in the separate field of audience reception studies. In these audience studies the focus is usually more on social interaction around one specific program or genre (such as soap opera), rather than a systematic analysis of the different genres that stimulate conversation or not, whether during the program itself, or afterwards [15]. Tovares for example analyzed conversations among family members and friends about 'Who Wants to Marry a Millionaire', a reality show [36]. An interesting study that does compare several genres is [20], but it looks at differences in attention style with regards to different genres, and not at communication patterns. The most comprehensive study we have found that studies communication patterns while watching television is [21], but also here several separate genres are singled out, and are not systematically compared.

In studies of social interactive television systems there is anecdotal evidence that genres of television or video content influence the way users do or do not talk, chat or otherwise interact with each other while watching television, as well as while socializing afterwards with friends or family (e.g. in [37]). Sport is often mentioned as one of those genres that are very well suited for stimulating social interactions. Harboe e.a. [18] evaluated a social iTV system that allows users to talk to each other over an audio link. In the focus groups as well as the field trials they conducted, participants agreed that sport programs are especially well suited for using their system. Also mentioned were cooking programs and certain (unspecified) movies. They conclude that when designing social iTV systems, the features should be optimized for the characteristics of sports viewing, although they acknowledge that other genres play a role too. Weldon [38] suggests creating an audio tool specifically designed for talking and shouting during a sports match, arguing that the voice chat to be implemented should be tailored to facilitate fan communication while watching a sports event.

However, sport is a genre that is very dependent on gender, being preferred more by males. Several studies have pointed out these differences, e.g. Brereton and O'Connor report that the three favorite genres of males in their study are comedy, sport and science fiction, while for the females this was comedy, soap opera and romance [3]. Similarly, Harboe e.a. [18] also mention that the women in their study would use their social iTV system more for programs other than sport. Another aspect that should cause us to be cautious about optimizing Social iTV systems for sports viewing, are cultural differences between the US and Europe (and other continents as well), and even within Europe between different countries. Cardoso [5] reports that sport as program genre is very popular in European countries such as Germany or Italy, in which it takes up $75 \%$ of the programs with the highest ratings (similar to US figures), whereas it is less popular in countries such as France or Belgium in which sport takes up less than $30 \%$ of the most popular programs.

A few studies of social iTV systems offer some more insight into the impact of different genres. Ducheneaut e.a. [12] asked their participants in an experiment where they watched television over a distance, what kinds of television programs they watched in groups. Genres with bursty rhythms, redundant content or people-centered 
content were considered as the most sociable. Schatz e.a. [30] studied a social interactive television system and observed and explicitly asked participants about the chat suitability of certain television genres. They observed that news and lifestyle TV significantly encouraged communication activity, which was also reflected in the ratings of chat suitability of both genres by their participants. Documentaries and user generated content from YouTube was rated rather low, to the surprise of the authors who assumed that personally created content might be more suited for social interaction. It is however unclear which program genres were included in this study, and what the scores of the other genres were. Shamma e.a. [32] have executed a preliminary study of Zync Messenger, a system that allows users to share a synchronized video and chat at the same time. They used the YouTube category of the shared videos in the study to track which genres were shared most. The top three of their most shared videos is music, comedy and entertainment. Unfortunately, although they do report differences in the amount of chat activity during different videos (e.g. longer videos have more chat activity), they have not correlated this with the program genre. As such, the top three list they show is very similar to the top three list of the most popular video categories on YouTube itself [17], and it does not offer us more insight in the sociability of these genres.

We report the results of a study carried out in Belgium with a Flemish, Dutch-speaking audience, offering a new perspective as well as a more systematic analysis of communication patterns surrounding certain television program genres and their impact on the design and evaluation of social iTV systems. We also distinguish two modes of interaction where television genres play a different role: synchronous interaction (talking while watching television) and asynchronous interaction (talking about television programs). Although the system used in this study is focused mainly on asynchronous interaction (sending annotated video clips to friends or family, who can watch it later), our results also allow us to discuss the impact of television genres on synchronous interaction.

\section{STUDY SETUP}

We performed a user test with the Ambulant Annotator system to gain insight in how people annotate and share video clips from the couch. The use of the system, in which clips from two documentaries and several film trailers were included that the participants could send and receive, provided the context for the questionnaires and group interviews which formed the basis of the analysis presented in this paper.

In this part, we will first give a description of the system that was used during the study, focusing on these aspects that are relevant for the discussion in this paper. Secondly, we will describe the setup of the user test.

\subsection{Summary of the System}

In order to evaluate the remote sociability of video sharing, a prototype system was designed and developed [7], [8]. The system provides functionality for sharing audiovisual content between users. It is composed of a number of architectural blocks including a home network that connects interactive devices and rendering components, the social network of the user, and a media database. The major innovations from the prototype system are:
- Distributed Rendering/Control of media: the system's architecture provides support for distributed rendering and control of media content. That is, the media can be rendered in a number of devices ranging from high-definition television sets to mobile phones. At the same time, the user can control (e.g., play, pause, fragment, enrich) the videos using a number of interactive devices, such as a mobile phone, synchronized with watching the media content.

- Media Enrichment: the user can fragment video material, so parts of it can be shared, and enrich it using other media overlays (e.g. audio commentary). Such fragmentation and enrichment is done using a high-level description language, so it does not modify the actual bits composing the video.

- Social Sharing: the system provides functionality to share the enriched media with other peers of the social network. The sharing mechanism can distribute the enriched material to mobile phones, television sets, or upload it to web pages and personal blogs.

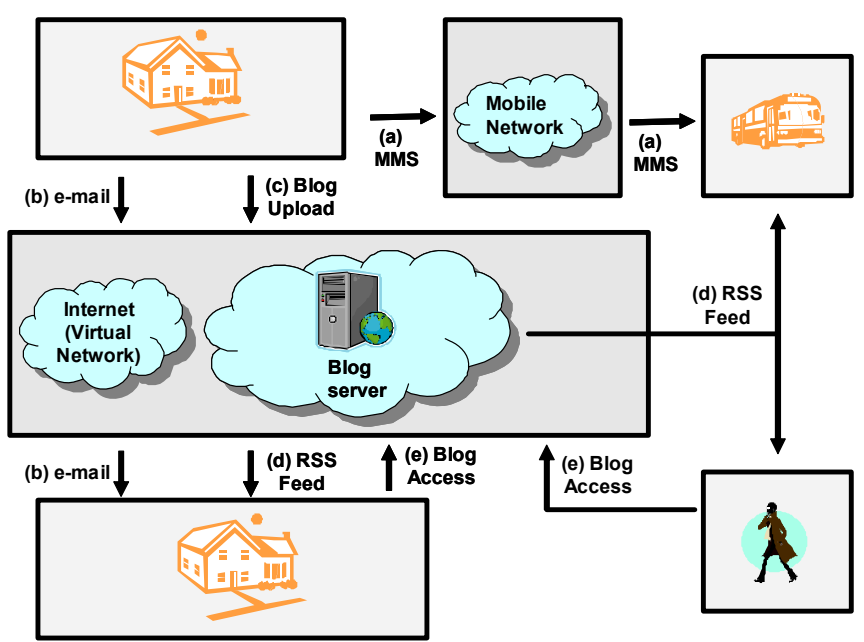

Figure 2: System's Architecture [8].

Figure 2 shows the basic architecture of the system, in which recommendation messages containing the enriched media can be shared using any of the existing network's infrastructures. The user might want to send a private email that contains a pointer to the enriched media or send it as an MMS to a mobile user. On the other hand, the user might want to share the media with the world by uploading it to his/her personal blog or social web site (e.g. Facebook). All such alternatives are considered in the architecture utilized for user testing.

The basic premise of our architecture is to consider media sharing as an incidental activity. The basic scenario is that a user, when watching audiovisual material, can fragment, enrich, and share it with his/her peers by using an extended remote control such as his/her mobile phone. An example of the media sharing interface is illustrated in Figure 3. For further information on the system software see [8], [7]. 


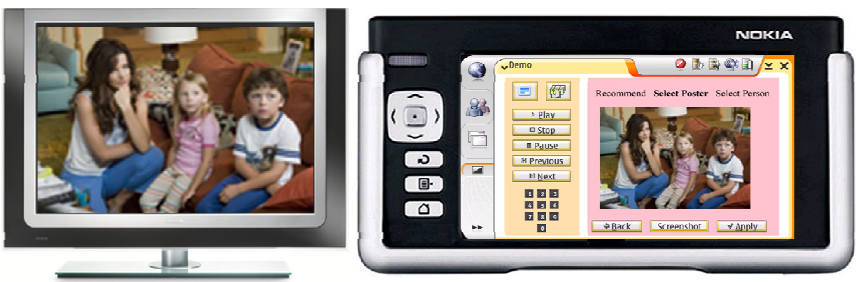

Figure 3: Sharing Interface [8]

\subsection{User test}

Twelve groups of users were recruited for the user test. One test session involved one single group, lasted for two hours, and was audio and video recorded. Each group consisted of two to five people that knew each other well, either as friends or as family members, and sometimes a mix of both. In total 36 participants took part in the test, with ages ranging from 14 to 72 (median 23). As for gender, 13 participants were male and 23 participants were female. Occupations ranged from students and housewives to public servants and teachers.

The test sessions took place in a simulated living room and consisted of four main parts: an explanation of the system, a collocated test situation, a remote test situation, and a group interview. After the second and third part, a couple of questionnaires were filled in by each participant individually. Each part of the test will be shortly explained below.

After greeting the participants, having them sign a consent form and giving a short briefing, the first part of the test was used for an extensive explanation of the system. This was done because the focus of the test was not on uncovering usability problems, but on how people would use the system and what their experiences with using the system were. Therefore, any usability issues in the system in development could be lowered by offering the users a thorough tutorial.

During the second part of the test, all members of the group stayed in the same room, and were asked to browse through the available content, select those items they wanted to share with someone they knew, clip and annotate these clips if they wanted to, and finally send them to someone they had to specifically name (the latter was done to add some realism to the test, so users would really think about which content to send to which person). Figure 4 shows the users of one test during the collocated test situation. After this part of the test, which generally lasted between 20 minutes and half an hour, a short questionnaire was filled in by each individual participant, to assess their first reactions to using the system.

In the third part of the test, the group was split into two subgroups. One sub-group (sometimes a single person) stayed in the simulated living room, whereas the other sub-group (or single person) was led to a separate room. For this part of the test, the participants in the living room were asked to edit and annotate clips to send to the participants in the other location. This way, the participants knew their edited clips were really received by someone. Also, the remote group could experience the different ways of receiving content (via PC, TV, SMS or a blog). Again, a short questionnaire was filled in by each single participant to collect their reactions on this part of the test (including their device preferences for receiving content), as well as a slightly longer questionnaire that covered the general use of the system and a final questionnaire about their daily media use (which included several questions on genre preferences).

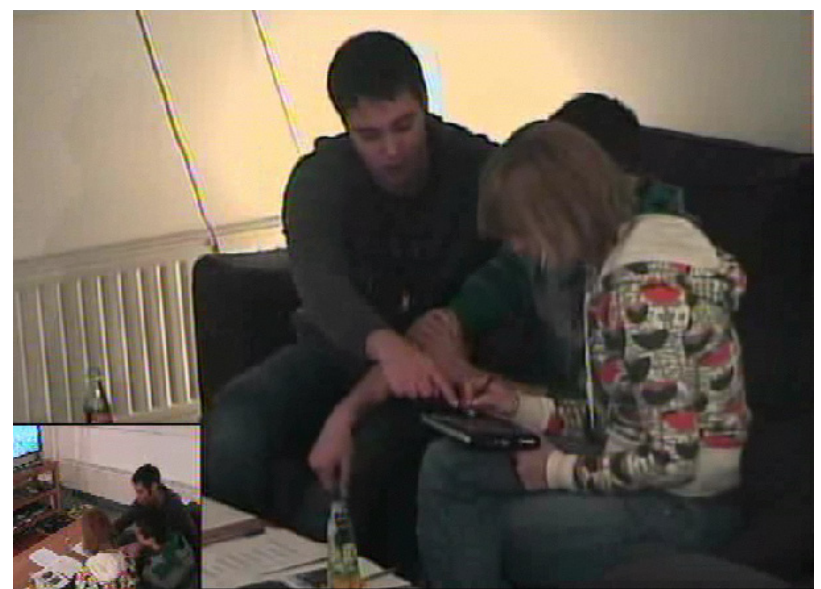

Figure 4: Users in the collocated test situation

Finally, the fourth part of the test consisted of a group interview lasting about twenty minutes, which covered several aspects such as reasons for sending clips, as well as device preferences. At the end of the test, users were rewarded with a gift coupon.

When analyzing the qualitative results, the video observations and participants' answers were coded and clustered using affinity diagramming. Only concepts that were observed in or mentioned by at least two groups were taken into account. Some issues were repeated by almost all groups, and were given more weight when interpreting the results. In the discussion below, sometimes quotes from single persons are used to illustrate a concept mentioned by several participants.

\section{RESULTS}

\subsection{Device preferences for receiving clips}

We asked the participants that received (and watched) video clips during the test via mobile phone, e-mail, blog or television, which of these methods they would prefer in the future. The other participants were not asked this question, because they did not have the experience of receiving video clips, and would therefore not be able to relate to the specificities of each device ${ }^{10}$ for receiving and watching video content. Participants were asked to rank the devices from 1 to 4 , where 1 is their most preferred device and 4 their least preferred device. In total, 15 participants answered this question, but 2 participants only gave one ranking for a single device (instead of a ranking for each device) so they were excluded from the results, leaving us with 13 participants. Figure 5 shows the distribution of the different rankings for each device.

\footnotetext{
${ }^{10}$ E-mail and blogs are not actually a device, but are both usually used on a PC. In the discussion of the results, we therefore use the term 'device' to refer to either blogs or e-mail on PC.
} 


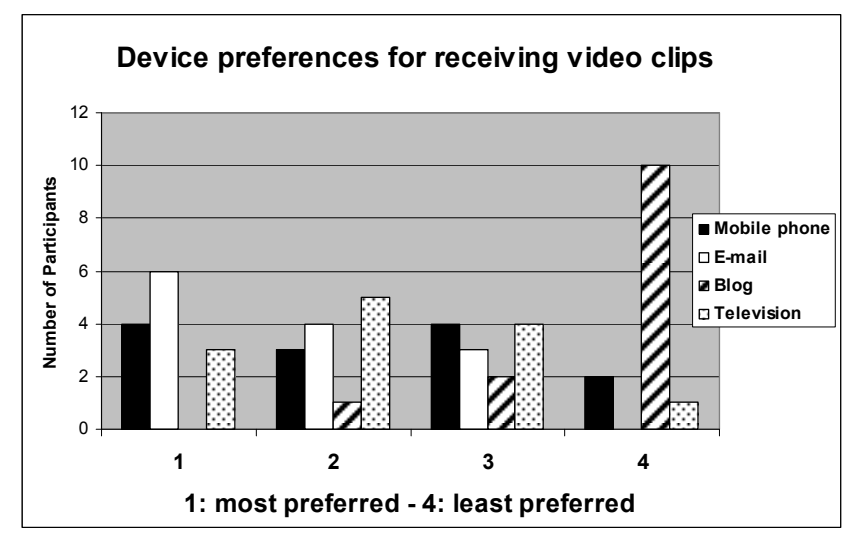

Figure 5: Device Preferences for Receiving Video Clips

Looking at the results, we can see that there are some trends, but not very outspoken. E-mail is mostly ranked first and television mostly ranked second, but differences are minimal and for mobile phone it is even more unclear, as it is ranked first, second and third by about the same amount of people. More outspoken is the last ranking for blog as a way to receive video clips. The reason for these mixed results can be found in the results of the qualitative analysis of the observations and group interviews. As the blog option was not discussed a lot during the group interviews, we will not include this in our following analysis.

During the group interviews after each test, the participants mentioned several factors that influence which device they prefer for receiving clips, which explains why the quantitative analysis showed mixed results. Clip length, content quality and immediacy were the three main factors that influenced the choice of device for receiving clips. Short clips that one would like to see immediately, without disturbing someone else, such as the weather forecast or breaking news, were preferred to be received by MMS on a mobile phone because you always have it with you. However, the size of the mobile phone screen was considered too small by most participants to watch something of higher quality (of content, not image quality), such as documentaries or movies. Some participants noted they would want to receive a notification on their phone, and then forward it to the device on which they would want to watch it later, such as their television or their PC. The size of the television screen, as well as the more relaxed and comfortable setting were mentioned by several participants as reasons to receive longer and higher quality content on TV.

Some participants would want to know if someone was watching TV at the moment before sending them a clip, which raises a fourth factor: privacy. Several participants indicated they would find it problematic that multiple people could be watching the clip they have sent, especially if it was intended for a specific person. One person said it would not be good if she sent something to her friend, and her friend's husband would be watching it, while another person said he would not send it to a girlfriend if he knew her family would be sitting there as well. Some participants said they would send annotations or clips that are very personal via email or text message rather than via TV, because they do not know who is watching. E-mail was considered by some participants as more private than $\mathrm{TV}$, having almost the same immediacy as a mobile phone (these participants indicated having their e-mail always open) and having a bigger screen than a mobile phone, allowing higher quality content to be comfortably viewed.
Table 1: Summary of Device Preferences According to Video Properties

\begin{tabular}{|l|c|c|c|c|}
\hline & Length & $\begin{array}{c}\text { Content } \\
\text { quality }\end{array}$ & Immediacy & Privacy \\
\hline $\begin{array}{l}\text { Mobile } \\
\text { Phone }\end{array}$ & Short & Low & High & High \\
\hline E-mail (PC) & Medium & Medium & Medium & High \\
\hline TV & Long & High & Low & Low \\
\hline
\end{tabular}

In summary, we can categorize the three most mentioned devices for receiving videos according to the properties of the videos in Table 1. A mobile phone is usually preferred for short clips with low content quality that can be watched in private, and that have an urgent character (high immediacy). E-mail received on $\mathrm{PC}$ is preferred for medium length clips of medium content quality, which may or may not have an urgent character but can also be watched in private. Finally, television is a device on which people would want to receive long, high quality clips without an urgent character that can be watched in company.

\subsection{Talking and sharing: the impact of different television genres}

The 36 participants in our study answered several questions about television genres, so we could get a good picture of a) during which program genres people talk most or least, b) about which program genres they talk most or least and c) which genres they would like to share with someone. Participants were allowed to indicate multiple genres. We created a list of 18 program genres (plus an open category 'others'), based on the "EBU system of classification of RTV programmes" [13], wherein programs are classified according to intention, format, content, target group, origination, language or participation. We chose the content classification, as this best reflected a variety in genres, including such formats as sitcoms, reality shows, the weather report, etc. but made a selection of the most popular genres according to TV program viewer ratings, otherwise the list would become too long. For each genre, we gave a couple of examples of popular television programs. The full list of genres we used is available on request.

In order to find patterns that would help us to understand the reason for preferring certain genres, we were especially interested to see if there were similarities between a) genres during which people talk while watching, b) genres people talk about (at work, at school, in the train, ...) and c) the genres they would like to share videos of. A first glance already showed some interesting results. If we rank the most often chosen program genres, we see similar genres in the list of genres during which people talk least (Figure 6), about which people talk most (Figure 7) and people would like to share (Figure 8 ). In other words, people would like to share videos with each other of program genres that are discussed at work or at school, but those are genres during which people usually do not talk while watching. Those genres are film, news, news magazines and documentaries. 


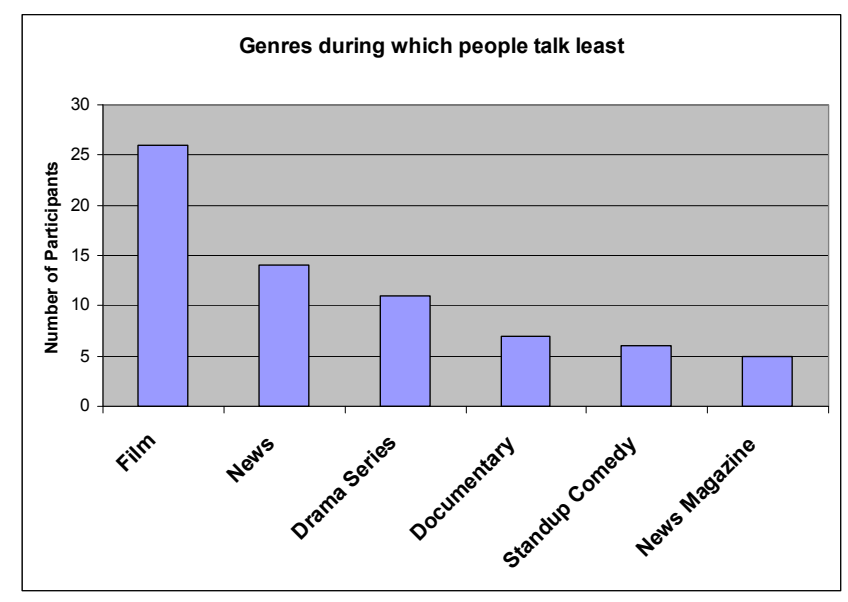

Figure 6: Genres during which people talk least

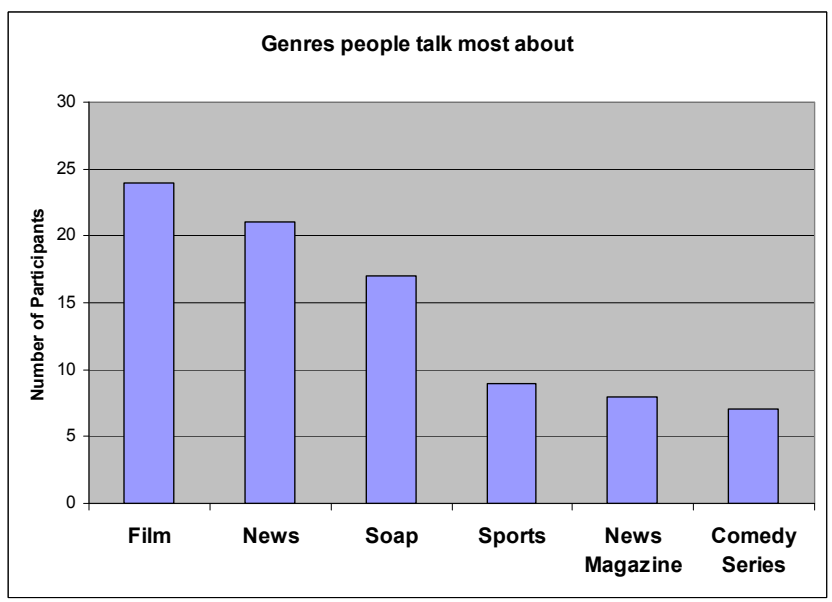

Figure 7: Genres people talk most about

Based on this first glance, we decided to look at correlations between items of the different lists, e.g. checking if it is usually the same persons that like to talk while watching and also want to share the video of a certain genre. However, we are not looking for predictive statements (which cannot be made on the current data alone) but rather detect which genres are the most 'sociable'.

When comparing the lists of genres our participants most talk about with the genres our participants would like to share, we see very strong positive correlations $(\mathrm{p}<.01)$ for the genres news, sport, soap opera, docusoap, reality show, talk show, comedy series and quizzes, as well as strong positive correlations $(\mathrm{p}<.05)$ for the genres film, animation film, standup comedy and music programs. Based on these correlations it is likely that most genres people talk about (12 out of 18) are also those genres people tend to share. On the other hand, for genres during which people talk most we find correlations with a preference for sharing for eight genres: debate programs, sport, soap, $(\mathrm{p}<.01)$, animation series, standup comedy, music programs or hobby programs $(\mathrm{p}<.05)$. Only sport and soap are genres that were scored by more than $25 \%$ of our participants, and thus are most relevant in our current discussion. In contrast, if we compare sharing certain genres with the list of genres during which people talk least, we find no significant correlations for any genre. Unsurprisingly, almost all genres that people have indicated as favorite genre positively correlate very strongly with genres they want to share, except for the weather report and talk shows. So if something is a favorite genre, people tend also like to send it.

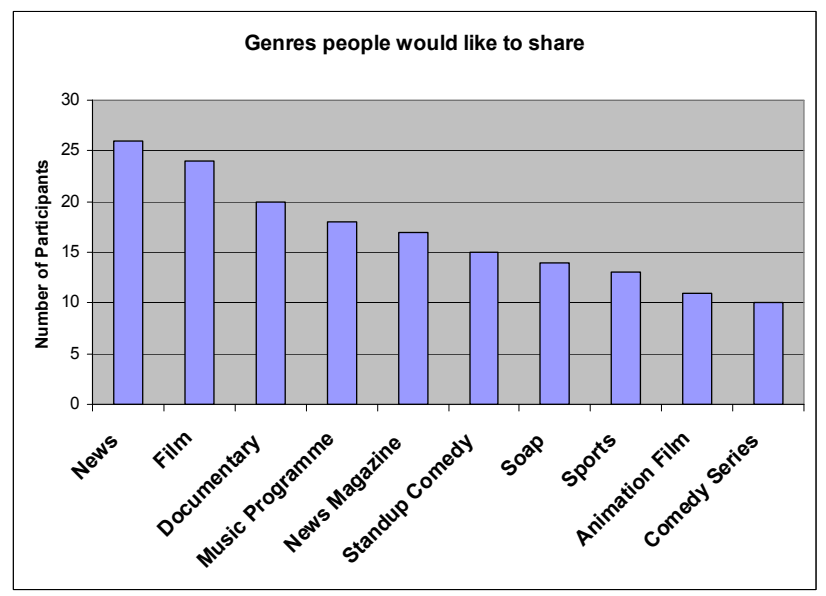

Figure 8: Genres people would like to share

Some genres show mixed results. News, for example, is indicated by almost as many people as a genre during which they prefer to talk (39\%), as during which they prefer not to talk $(50 \%)$. There is a very strong negative correlation between the two $(\mathrm{r}=$ $0.67, \mathrm{p}<.001$ ), so these are actually different people, in total $89 \%$ of our participants. Statistical analysis on gender or age differences showed no evidence that could explain the occurrence in both lists.

On the other hand, soap opera is a genre that people not only talk about afterwards, but also while watching $(\mathrm{r}=.657, \mathrm{p}<.01)$. While this is also the case for sport, news magazines or docusoaps (the two questions positively correlate with $\mathrm{p}<.01$ ), $47 \%$ of our participants talk during soaps as well as about soaps. For sport (the next runner-up), only 25\% talk during sport and 30\% about sport. Interestingly, sport and soaps are the genres that have strong positive correlations between talking during watching and sharing. Although these two genres are each known to be favored by members of a specific gender [3], we did not find significant gender differences in our data (maybe because gender was not well spread in our sample).

Finally, we can look at the list of genres during which people talk most (Figure 9), as an indication of which genres are especially suitable for a synchronous social iTV system. Although sport is mentioned by 11 participants $(31 \%)$, we see that several other genres are even more popular to talk while watching, such as news $(50 \%)$, soap operas $(47 \%)$ and quiz shows $(33 \%)$. Reality shows $(28 \%)$ and talk shows $(25 \%)$ also rank very high.

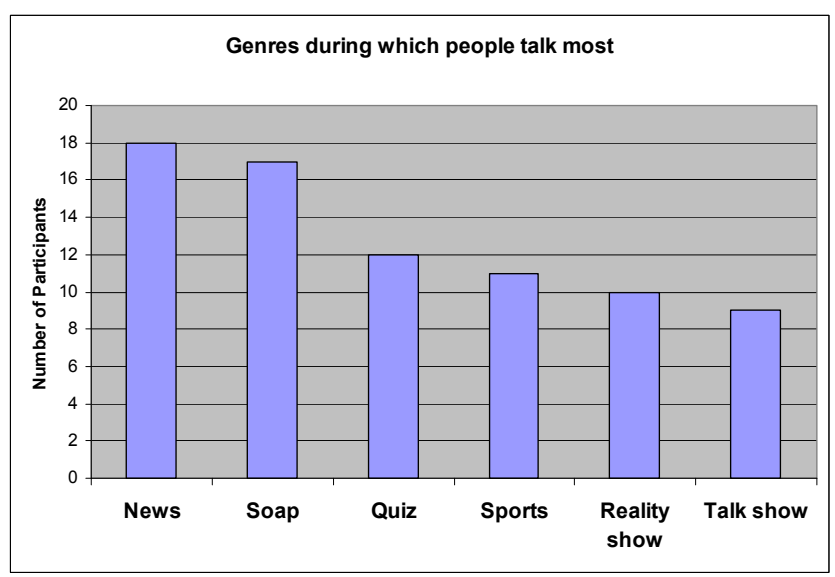

Figure 9: Genres during which people talk most 


\section{DISCUSSION}

As discussed in the related work section, video sharing systems can benefit from connecting several devices, so the question if and how genres play a role here is important as well. Although we did not explicitly ask for it in the interviews about device preferences, the genre of the clips our participants wanted to send or receive was often mentioned spontaneously. Documentaries and movies were often mentioned as 'higher quality content' which is preferably viewed on television, in contrast to the weather or breaking news, which people prefer to watch on their mobile phone. This confirms previous research that states that although the PC is becoming more and more an entertainment system, allowing online video watching (e.g. on YouTube) or even chatting while watching television (e.g. on Joost), the living room is still seen as the most comfortable viewing location, because of the big television screen and comfortable couch [3]. When compared with the results on genre preferences, documentaries and movies are genres during which one usually does not talk while watching, whereas one does talk during watching news and (to a lesser extent) the weather. Even though these similarities are anecdotal and not comparable in a systematic way, they do indicate that the two aspects of this paper, device and genre preferences, are linked to each other.

Next, we would like to look for reasons behind certain preferences, and especially the special cases we found in the data. Let us first look at the - at first sight - contradictory results about the news genre. Almost the same amount of people that would not talk during the news, would talk during the news. Although this could point at personal preferences, there are no other genres that show such contradictory results. Part of the answer might be found in a similar study about television attention styles (i.e. the length and frequency of looks at television) by Hawkins e.a. [20], which is closely linked to communicating while watching [16], [37]. Hawkins e.a. found that, contrary to what they expected, attention styles were not a steady characteristic of an individual, but differed according to genre. Even specific gratifications or favorable attitudes for each genre accounted for little of the variation in attention style. This could indicate that communication patterns are also much more dependent on genre rather than on individual characteristics or genre gratifications and preferences. Thus, the variation in news should be sought elsewhere. An important element in attention style mentioned by Hawkins e.a. is the relative importance of plot in the viewing experience. Dramas and movies ask for more continuous attention than commercials and news items. As Hawkins e.a. point out, news magazines are an anomalous case, having a more narrative and plot-like structure, but with short items of less than 15 minutes. European news broadcasts might be more comparable to this structure, as the news in Europe is typically less fast-paced than in the US. This could partly explain the mixed results of the news genre in our questionnaire, because certain items in the news are longer and contain more plot than other parts of the news.

With the importance of a plot structure in mind, it is easier to see a pattern emerging in the genre preferences of our participants. All genres in the top six of those genres during which people do not talk while watching contain a plot-structure that calls for their full attention, e.g. film, drama or documentary. Reversely, the top six of genres during which people do talk have less plot-structure, such as quiz, sport or reality show (keeping in mind the ambiguous nature of news). Linking this again with the results on device preferences, we can argue that our category of 'content quality', referred to like this by our participants, is rather related to the plot structure of the content. So, genres with more plot structure are preferred to be watched on television, whereas genres with less plot structure can be watched on a mobile phone.

Another odd one out in our genre preferences is soap opera, during which people talk while watching, but also talk about afterwards. This is the same for sport (although for fewer participants). We can argue that plot structure is less important in soap operas as it focuses more on the situation at hand rather than the bigger plot (which explains why it is so easy to start watching a soap after missing a couple of episodes) as well as in sport. But although most genres in our study are either in the list of genres during which people talk most or in the list of genres people talk most about, soap opera and sport are in both lists. When looking for an explanation for this discrepancy, we have to dig deeper in the social roles certain genres play. According to Lull [25], citing others as well, soap opera is often used by viewers to learn how to deal with relational conflicts, and discuss this with friends and relatives. This explains why people use this in daily conversations, as evidenced by several researchers (see e.g. [15]). A similar argument can be made for reality shows [12]. Other genres however show other patterns. People often talk during quiz shows for example, because viewers like to show off their knowledge to each other [25], but do not often talk about quiz shows. Showing competence during a quiz show is usually done synchronously, so one can answer a question before a contestant, and not by bragging afterwards how many answers you knew.

To conclude our discussion, Figure 10 summarizes the relationship between the different aspects discussed in the paper. Although plot structure can account for most of the preferences in talking about and sharing videos, for some genres the relationship is more complex and related to some of the social uses of television. More research would thus be needed into this interplay between these social uses and specific genres.

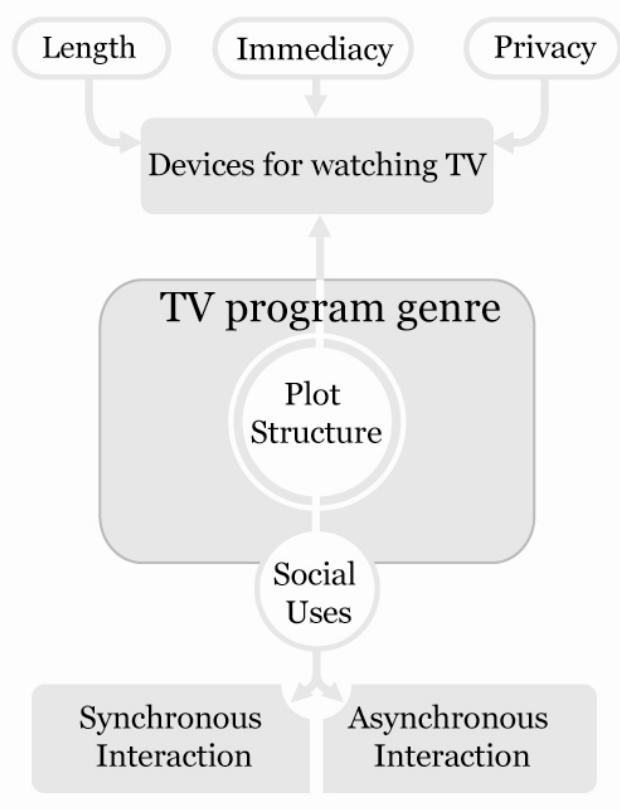

Figure 10: The role of television program genres 
Although the study we performed offered important insights into device and genre preferences, there are a couple of limitations. First of all, the genres used during the test were trailers (from films) and (nature) documentaries. However, the most mentioned genre for sharing is news, which was not available in the system, so this influence might be minimal. Another limitation is the fact that these results are based on a lab study, with self report questionnaires, and not a longitudinal study in a real environment observing actual conversation patterns during and after watching television. However, until such a longterm study is conducted, this study gives some first insights in different genres and device preferences as well as their impact on the design and evaluation of social iTV applications. Finally, the number of participants in this study is rather limited, so the statistical results should be interpreted with caution.

\section{CONCLUSION}

News, soap, quiz and sport are those genres during which our participants talk most while watching. This suggests that for designing features for synchronous social iTV systems, these genres are most suitable. For asynchronous systems (such as for video sharing or leaving notes on top of a video stream), film and news are the two genres people talk most about and which they would also like to share, while documentaries and music programs also score high. This suggests that the focus of asynchronous TV systems should be on these genres when designing features for these systems. However, we also found many subtle differences between different devices and genres that brought up several new questions and warrants further research on this topic. We believe this and future research on this topic is important for the further development of social iTV systems for several reasons.

First of all, the results of this study can be used for evaluating social iTV systems. If we can take into account the 'sociability' of TV genres as well as user preferences for devices, either for synchronous communication or for asynchronous sharing, we know what genres are important indicators of the success of a system.

Secondly, we have shown that sport might not be the only genre to optimize the design of social iTV systems for. Systems such as AmigoTV [9] and Motorola's SocialTV [18] contain cartoons or prefabricated messages. These tools should take into account a wider array of genres. Instead of including all possible genres, they could be focused on these genres during which people talk most. Similarly, television might not be the only device to be part of a social iTV system, and other devices such as the PC or mobile phones could be part of the 'networked TV' environment as well.

Thirdly, when setting up lab tests or field trials, sometimes certain video content has to be provided when it is not possible to use a broadcast stream. These results can help in deciding which genre of content to provide in the system (see e.g. [12]).

Finally, certain design choices can be made based on the results of this study. For example, during several user tests of social iTV systems (e.g. [16]) we have seen that a 'do not disturb' status is important for people when watching television. However, due to the lean-back nature of television, users are not inclined to change their status each time they do or do not want to be disturbed. If we know during which genres people do not want to talk, we can design the system to automatically change the user's status to 'do not disturb' for these genres.

\section{ACKNOWLEDGMENTS}

We would like to thank Kenny Verbeke, Wim Konings and Christof van Nimwegen for their help. CUO conducted this work within the IBBT ISBO-VIN project, funded by the Flemish Government. CWI conducted this work within the IST-FP7 project Ta2 (214793), partly funded by the EU.

\section{REFERENCES}

[1] Bernhaupt, R., Obrist, M., Weiss, A., Beck, E., and Tscheligi, M. 2007. Trends in the living room and beyond. In Proceedings of EuroITV, 146-155.

[2] Bignell, J., 2003. An Introduction to Television Studies. New York: Routledge

[3] Brereton, P. and O'Connor, B. 2007. Pleasure and Pedagogy: The Consumption of DVD Add-Ons Among Irish Teenagers. Convergence, 13(2), 143-157

[4] Bulterman, D.C.A., Cesar, P., and Jansen, A.J. 2006. An Architecture for Viewer-Side Enrichment of TV Content. In Proceedings of the ACM Multimedia Conference, 651-654

[5] Cardoso, Gustavo, 2006. The Media in the Network Society: Browsing, News, Filters and Citizenship, Lisboa, Portugal. CIES - Centre for Research and Studies in Sociology

[6] Cesar P., Bulterman, D.C.A., and Jansen, A.J. 2008. Usages of the Secondary Screen in an Interactive Television Environment: Control, Enrich, Share, and Transfer Television Content. In Proceedings of EuroITV.

[7] Cesar, P., Bulterman, D.C.A., Geerts, D., Jansen, A.J., Knoche, H., and Seager, W., 2008. Enhancing Social Sharing of Videos: Fragment, Annotate, Enrich, and Share. Submitted to ACM Multimedia Conference.

[8] Cesar, P., Bulterman, D.C.A., and Jansen, A.J., 2007. Social Sharing of Television Content: An Architecture. In Proceedings of ISM2007 (Workshops), 145-150.

[9] Coppens, T., Handekyn, K., and Vanparijs, F., 2005. AmigoTV: A Social TV Experience Through Triple-Play Convergence. White Paper, Alcatel.

[10] Cruickshank, L., Tsekleves, E., Whitham, R., Hill, A., and Kondo, K. 2007. Making interactive TV easier to use: Interface design for a second screen approach. The Design Journal $10(3)$.

[11] Dearman, D., and Pierce, J.S. 2008. It's on my other computer!: computing with multiple devices, In Proceedings of CHI, 767776 .

[12] Ducheneaut, N., Moore, R.J., Oehlberg, L., Thornton, J.D., and Nickell, E. 2008. Social TV: Designing for distributed, sociable television viewing. In International Journal of HumanComputer Interaction, 24(2), 136-154

[13] European Broadcasting Union. 2007. Escort: EBU System of Classification of RTV Programmes. Technical report.

[14] Fallahkhair, S., Pemberton, L., and Griffiths, R. 2005. Dual device user interface design for ubiquitous language learning: Mobile phone and interactive television (iTV). In Proceedings of the IEEE International Workshop on Wireless and Mobile Technologies in Education, 85-92.

[15] Fiske, J., 1989. Television Culture. New York: Routledge 
[16] Geerts, D. 2006. Comparing voice chat and text chat in a communication tool for interactive television. In Proc. NordiCHI 2006, 461-464.

[17] Gill, P., Arlitt, M., Li, Z., and Mahanti, A. 2007. Youtube traffic characterization: a view from the edge. In Proceedings of the 7th ACM SIGCOMM Conference on internet Measurement. ACM, New York, NY, 15-28

[18] Harboe, G., Massey, N., Metcalf, C., Wheatley, D., and Romano, G. 2008. The uses of social television. ACM Computers in Entertainment (CIE), Volume 6 Issue 1

[19] Harrison, C., Amento, B., 2007. CollaboraTV: Using asynchronous communication to make TV social again, In Adjunct Proceedings of EuroITV2007, 218-222.

[20] Hawkins, R.P., Pingree, S., Hitchon, J., Radler, B., Gorham, B., Kahlor, L., Gilligan, E., Serlin, R.C., Schmidt, T., Kannaovakun, P., and Kolbeins, H. 2005. What produces television attention and attention style?: Genre, situation, person, and media perceptions as predictors. Human Communication Research. 31, 162-187

[21] Holly, W., Püschel, U., and Bergmann, J. (eds.) 2001. Der sprechende Zuschauer. Wie wir uns Fernsehen kommunikativ aneignen. Wiesbaden: Westdeutscher Verlag

[22] House, N.V., Davis, M., Ames, M., Finn, M., and Viswanathan, V. 2005. The uses of personal networked digital imaging: an empirical study of cameraphone photos and sharing. In extended abstracts on Human factors in computing systems, 1853-1856.

[23] Kindberg, T., Spasojevic, M, Fleck, R., and Sellen, A. 2005. I saw this and thought of you: some social uses of camera phones, In extended abstracts on Human factors in computing systems, 1545-1548.

[24] Knoche, H., McCarthy, J.D., and Sasse, M.A. 2005. Can small be beautiful?: assessing image resolution requirements for mobile TV. In Proceedings of the ACM international conference on Multimedia.

[25] Lull, J. 1980. The social uses of television. Human Communication Research, 6, 197-209

[26] Mate, S., Chandra, U., and Curcio, I.D.D., 2006. Movablemultimedia: session mobility in ubiquitous computing ecosystem, In Proceedings of the International Conference on Mobile and Ubiquitous Multimedia.

[27] O'Hara, K., Mitchell, A. S., and Vorbau, A., 2007. Consuming video on mobile devices, In Proceedings of CHI2007, 857-866.
[28] Park, J., Blythe, M., Monk, A., and Grayson, D. 2006. Sharable digital TV: relating ethnography to design through unuseless product suggestions. In extended abstracts on Human factors in computing systems, 119-1204.

[29] Repo, P., Hyvönen, K., Pantzar, M., and Timonen, P. 2004, Users Inventing Ways to Enjoy New Mobile Services -- The Case of Watching Mobile Videos. In Proceedings of the Annual Hawaii International Conference on System Sciences, Track 4, p.40096.3.

[30] Schatz, R., Baillie, L., Fröhlich, P., and Egger, S., 2008. Getting the Couch Potato to Engage in Conversation: Social TV in a Converging Media Environment. In: Adjunct proceedings of EuroITV2008.

[31] Seager, W., Knoche, H., and Sasse, M.2007. TV-centricity requirements gathering for triple play services. In Adjunct Proceedings of EuroITV, 274-278.

[32] Shamma, D. A., Bastea-Forte, M., Joubert, N., and Liu, Y. 2008. Enhancing online personal connections through the synchronized sharing of online video. In CHI '08 Extended Abstracts on Human Factors in Computing Systems. ACM, New York, NY, 2931-2936

[33] Shamma, D.A., Shaw, R., Shafton, P.L., and Liu, Y. 2007. Watch what I watch: using community activity to understand content, In Proceedings of the international workshop on multimedia information retrieval, 275-284.

[34] Södergård, C. 2003. Mobile television -- technology and user experiences. Report on the Mobile TV project, VTT Publications.

[35] Taylor, A.S., Harper, R. 2002. Age-old practices in the 'new world': a study of gift-giving between teenage mobile phone users, In Proceedings of the SIGCHI conference on Human factors in computing systems, 439-446,

[36] Tovares, Alla V. 2006. Public medium, private talk: Gossip about a TV show as 'quotidian hermeneutics'. In: Text \& Talk 2006, 26(4/5), 463-491

[37] Weisz, J. D., Kiesler, S., Zhang, H., Ren, Y., Kraut, R. E., and Konstan, J. A. 2007. Watching together: integrating text chat with video. In Proceedings of the SIGCHI Conference on Human Factors in Computing Systems CHI '07. ACM, New York, NY, 877-886

[38] Weldon, M. 2006. Audio tools for sports fan interaction. In CHI '06 Extended Abstracts on Human Factors in Computing Systems CHI '06. ACM, New York, NY, 1511-1516 